Running Head: Individual Differences in Brain Development

This is the author version of an article published in Biological Psychiatry. The final authenticated version is available online at: https://doi.org/10.1016/j.biopsych.2020.01.027

\title{
Modeling Individual Differences in Brain Development
}

\author{
Andrik I. Becht ${ }^{1,2}$, Ph.D. \\ Kathryn L. Mills ${ }^{3}$, Ph.D.
}

${ }^{1}$ Brain and Development Research Center, Developmental and Educational Psychology, Leiden University, Leiden, The Netherlands

${ }^{2}$ Research Center Adolescent Development, Utrecht University, Utrecht, The Netherlands ${ }^{3}$ Department of Psychology, University of Oregon, Eugene, OR, USA

\author{
Corresponding Author: \\ Kathryn L. Mills \\ Department of Psychology \\ 1227 University of Oregon \\ Eugene, OR 97403 \\ klmills@uoregon.edu
}

keywords: adolescence; heterogeneity; longitudinal; MRI; mental health; neuroimaging 4,035 words 


\begin{abstract}
Within the field of developmental cognitive neuroscience there is an increasing interest in studying individual differences in human brain development in order to predict mental health outcomes. So far, however, most longitudinal neuroimaging studies focus on group-level estimates. In this review, we highlight longitudinal neuroimaging studies that have moved beyond group-level estimates to illustrate the heterogeneity in patterns of brain development. We provide practical methodological recommendations on how longitudinal neuroimaging datasets can be used to understand heterogeneity in human brain development. Finally, we address how taking an individual-differences approach in developmental neuroimaging studies could advance our understanding of why some individuals develop mental health disorders.
\end{abstract}


Half of all lifetime mental health disorders have their onset by the mid-teens $(1,2)$. A major goal of neurodevelopmental research has been to understand how the brain develops in individuals who are diagnosed with mental health disorders, and to use this information for prevention and prediction efforts (3). Much work has been motivated by the premise that identifying atypical patterns of brain development in individuals diagnosed with mental health disorders will uncover the biological processes underlying these disorders $(4,5)$. Further, through knowing when these deviations in brain development occur, researchers might identify the optimal window of opportunity during development when interventions are likely to be most effective, or target intervention efforts for processes that are changing most during these periods of development. Indeed, there is increasing interest in using information about an individual's brain development to predict mental health outcomes, and to understand what individual or social environmental factors impact the brain's development in a way that might increase the risk for developing a mental health disorder. To reach these goals, we must shift from a focus on group-level models of brain development to approaches that characterize the heterogeneity that is present between individuals within the developing brain. Thus, with the term heterogeneity, we refer to individual differences in developmental trajectories of the brain. The main goals of this non-exhaustive review are to 1) highlight empirical longitudinal neuroimaging studies that have moved beyond group-level estimates, using methods that may aid in our understanding why some individuals develop mental health disorders whereas others do not, and 2) provide methodological recommendations for future studies interested in characterizing individual differences in patterns of brain development.

\section{Individual Differences in Longitudinal MRI Studies}


Since the 1990s, researchers have examined longitudinally acquired magnetic resonance imaging (MRI) data to characterize the shape of development in brain structure and function. There are now several datasets of individuals with multiple MRI scans spanning childhood and into young adulthood, including large datasets that are publicly available for researchers to use for their own investigations (e.g., Nathan Kline Institute Rockland; NIH MRI Study of Normal Brain Development). One of the most important advantages of longitudinally acquired data compared to cross-sectional data is the ability to characterize change within an individual, as well as the ability to study how individuals differ from each other in their developmental trajectories (6). Besides, age heterogeneous cross-sectional samples face the problem of potential cohort effects that may limit the validity of estimated age effects (7-9). Beyond the general reasons why longitudinal data are needed to answer questions about development, the large individual variability present at any age in both brain structure and function hinders drawing developmental conclusions about neural processes when datasets contain solely one measurement per person (Figure 1). For instance, the wide variability in cortical thickness in healthy developing children makes it impossible to draw the conclusion that children with lower cortical thickness compared to their same aged peers experience "premature thinning of the cortex," although this incorrect interpretation continues to persist (10).

To identify those individuals who demonstrate aberrant brain development, such as premature cortical thinning, it is vital to move beyond cross-sectional estimates and examine individual developmental trajectories. In doing so, it is possible to examine whether those individuals with relatively lower cortical thickness at baseline also show accelerated cortical thinning (which can also be conceptualized as a faster rate of change) across time compared to individuals who start with higher levels of cortical thickness (Figure 2). In addition to describing heterogeneity in developmental trajectories, individual differences in 
neurobiological developmental trajectories may also be used for predictive purposes (3). However, if the goal is to predict an outcome, individuals need to vary from each other. That is, if a measure (e.g., cortical thickness) was identical across all participants, it would have no predictive value. Similarly, individual differences in within-individual change over time (e.g., rates of cortical thinning) are necessary in order for these measurements to be predictive.

Thus, two questions remain. Do individuals differ in the within-individual change? And, can we use individual differences in neurobiological developmental trajectories to predict mental health outcomes?

So far, however, most longitudinal MRI studies have focused on establishing grouplevel trajectories of brain development. Many of these studies describe the average shape of development, often by comparing linear functions to non-linear functions. For instance, a number of structural brain imaging studies present different group-level trajectories across the cortex, between cortical and subcortical regions, between surface area and cortical thickness, or across sex from childhood into adolescence or young adulthood (11-15). See (16) for an extensive overview of previous longitudinal studies on structural brain development. While many of these studies reveal differential patterns of development across brain measures or regions, very few examine heterogeneity in the developmental patterns between-persons. And although structural brain development has been investigated with longitudinal MRI for over 20 years, replicable models have only been established in within the past few years (17-19). These recent studies have demonstrated substantial variance between individuals in the overall size of brain structures; for example, overall cortical volumes of neurotypically developing individuals can be $70 \%$ the size of same-aged peers (Figure 1a). Although there is less inter-individual variability in cortical thickness compared to surface area in the developing brain across participants (19), it is important to note that 
variability in these measures does not wane across development, and is present across childhood and into young adulthood.

Inter-individual variability within the developing brain can be even greater for estimates of functional activation and connectivity derived from MRI. While not as abundant as structural MRI studies of brain development, over the past years there have been an increasing number of longitudinal task-based and resting-state functional MRI studies spanning childhood and adolescence (see 20 for recent review). These studies illustrate considerable variability in the task-based MRI measures of neural responsivity to a variety of tasks across childhood and into young adulthood (Figure 1b; (21-23). Further, longitudinal examinations of resting state functional connectivity MRI also demonstrate individual variability of connectivity estimates in childhood and across adolescence (Figure 1c; (2426)). The substantial individual variability present across development in functional MRI measures demonstrates the necessity of collecting longitudinal data to establish individuallevel developmental patterns of brain activity and connectivity.

Although a limited number of studies have graphically illustrated the proportion of individuals that increase, decrease or remain stable in structural brain development (27-29), most longitudinal investigations have not formally tested the variability of developmental change. Variability in change is indexed by individual differences in the slope and can be observed in any study that plots longitudinal data of individual participants (Figure 1). The individual variability in slope magnitude and direction appears to be similar across cortical lobes for measures of cortical thickness, surface area and volume (19). However, subcortical and more spatially-specific cortical regions do demonstrate increased inter-individual variability in volumetric change across adolescence $(30,31)$. Compared to longitudinal investigations of structural brain development, it is more common to observe more interindividual variability in the direction and magnitude of developmental changes (slopes) 
observed from functional MRI task-based activation or resting-state functional connectivity (see examples in Figure 1). Some of these studies did examine, but not show significant variability in change $(21,32-34)$. However, these studies included two time points, which limits the power to detect significant slope variability $(35,36)$. Thus, to what extent these apparent stronger individual differences also reflect variability in growth remains an open question. We suggest that a critical next step is to directly test for heterogeneity in the patterns of brain change across development and examine whether and how these heterogeneous developmental patterns can be predicted by particular variables or predict particular outcomes. To this end, we consider a particular set of latent growth curve statistical models (e.g., 37-39) to examine these questions on heterogeneity in development.

\section{Modeling Heterogeneity in Brain Development}

To be able to examine a broad age range in a shorter time span, many longitudinal MRI studies employ an accelerated longitudinal (cohort-sequential) sampling design, rather than a single-cohort design (40). Several existing accelerated longitudinal studies of structural and functional brain measures across development include at least three timepoints of data. Although more than three time points would further increase model flexibility and precision of growth parameters (41), modeling longitudinal data with three time points does allow for different growth trajectories (linear and non-linear) to be compared against each other to identify an average growth model for the dataset.

While the power to detect individual differences in developmental trajectories in accelerated longitudinal studies is largely dependent on the number of participants and total measurements $(40,42)$, some sufficiently powered accelerated longitudinal MRI studies have modeled individual differences in brain development. We outline three of these studies below as examples of approaches that can be used in future studies. We start with a study that 
informs on the first important question: whether individual differences in development are actually present. Next, we continue with two studies that illustrate how to predict these individual differences or predict an outcome from these individual differences in brain development. Specifically, the first study examines individual differences in the rate of within-individual change over time by modeling random intercepts and slopes in a linear mixed-effects model on three-wave longitudinal data (43). In a linear mixed-effects model (also referred to as multilevel modeling, or random effects modeling), the fixed effects capture the average starting point (or intercept) and average change (slope) for the whole sample. Random effects capture the individual differences in these starting points (i.e., referred to as a random intercepts) and changes over time (referred to as random slopes; see $(37,44)$ for an overview of growth curve modeling techniques). It is important to note that the intercept can be placed anywhere and is not necessarily always at the starting point of a timeline.

Applying these mixed-effects models on structural MRI, Tamnes and colleagues (2018) investigated individual differences in the development of hippocampal subregions using three waves data from the Braintime study. They found that while the cornu ammonis (CA1) decreased in size on average across childhood and adolescence, the inter-individual differences in the slope of change was substantial enough that including a random linear slope improved the overall model of this hippocampal subregion's development. By simply examining if the inclusion of a random slope for each participant improved their overall developmental model, Tamnes and colleagues were able to empirically test the presence of inter-individual variability in the developmental pattern of their brain measure of interest. This is one example of a strategy that can be readily adopted in future longitudinal investigations of brain development with at least three time points. 
The second study was one of the first task-based developmental functional MRI study with three time points to demonstrate the importance of moving beyond the study of individual differences in intercepts (22). They also applied a mixed-effects model to examine heterogeneity in growth of neural activity to understand cognitive developmental processes. This longitudinal study included individuals (aged 9-26 years) who participated between one and six waves and examined heterogeneity in developmental trajectories of brain activation in motor control, executive control, and error-processing regions. While activity in motor control regions showed no general developmental pattern across ages, individual differences in the developmental change (slope) were present but decreased in magnitude over time. Thus, despite different starting points in neural activity in several regions (modelled with a random intercept), individuals converged to a similar magnitude over time. These findings support increasing brain maturation for some regions in which individuals develop towards a similar endpoint (convergence) across time. Next to modeling individual differences in slopes over time, Ordaz et al. (22) examined whether these individual differences in development could be predicted by sex and IQ. They found that sex could explain part of the variability in development in some brain regions, primarily those regions involved in motor control. This study is another example of ways to apply growth modeling techniques to test for individual differences in development which could further inform theories on brain development.

Third, in one of our own studies, we applied latent growth curve models to three-wave longitudinal structural MRI data to model heterogeneity in the development of prefrontal cortical volume and nucleus accumbens volume. In addition to examining heterogeneity, we tested whether we could use the random slopes of prefrontal cortical volume and nucleus accumbens to predict individual differences in the formation of a strong sense of identity (45). Identity formation was operationalized with three dimensions: commitment to goals, exploration in-depth, and reconsideration of goals. To develop strong identity commitments, 
adolescents need to show information seeking behaviors and goal directedness to explore different identity alternatives and form strong commitments (46). Therefore, we expected that individual differences in structural brain development in regions that are involved in information seeking behaviors (i.e., prefrontal cortex), and goal-directedness (i.e., nucleus accumbens) would predict the formation of identity commitments over time as well. First, we modeled developmental trajectories of prefrontal cortical volume and nucleus accumbens volume and found that, on average, the prefrontal cortical volume decreased across adolescence whereas the nucleus accumbens volume remained stable. Second, we moved beyond mean-level developmental trajectories and found that individual differences in the overall size (captured with a random intercept) and the rate of developmental change (captured with a random slope) in the volume of these brain regions across adolescence predicted individual differences in identity development at a fourth time point. Specifically, adolescent participants with higher prefrontal cortical and nucleus accumbens volumes relative to their peers reported stronger commitments to goals, more in-depth exploration, and less reconsideration of goals. Moreover, adolescents who showed a less steep decrease in grey matter volume in these regions reported stronger commitment to goals and more indepth exploration. These results demonstrate the possibility of applying latent growth curve modeling techniques to examine whether individual differences in developmental trajectories in brain regions of interest capture relevant variability to predict certain behavioral outcomes (in this case, identity development) over time. While this latter study did not focus on predicting developmental psychopathologies (such as depressive or anxiety symptoms) specifically, the approach adopted in this study suggests the utility of moving beyond grouplevel estimates to predict individual differences in the development of behavior from brain features. 
Although we have to be careful in drawing definite conclusions from the three highlighted longitudinal studies, they illustrate that mixed-effects models and latent growth curve models can both be used to model average growth trajectories as well as heterogeneity in these trajectories. Importantly, mixed-effects models and latent growth models have similarities and differences in their applications. First, mixed-effects models and latent growth curve models are mathematically similar (e.g., 37). Second, both models are able to capture individual differences in within-person change (with random intercepts and random slopes). Yet, in contrast to mixed-effects models, latent growth curve models are highly flexible by allowing researchers to model average change and use individual differences in the rate of change (e.g., in structural brain development) as predictors of other variables of interest, all within the same model. In doing so, latent growth models make it possible to examine linkages between variability in brain development and behavioral development. Testing these brain-behavior linkages is considered an important next step to investigate to what extent individual differences in brain development capture meaningful variability (3). While latent growth models are able to capture individual differences in functional and structural brain development, these models assume that all individuals are drawn from the same homogeneous population, and that the developmental trajectory of each individual can be adequately described with a single estimate of growth parameters, around which an individual can vary (38). As such, latent growth models assume that all individuals develop in the same direction (47). This can be a problematic assumption, given that diverging patterns in the direction of changes can be expected for studies of task-based functional MRI and resting-state functional connectivity MRI.

\section{Methodological Recommendations and Future Directions}


Neurodevelopmental researchers who want to relax the assumption of homogeneity could apply mixture models (latent class growth analyses, LCGA; or growth mixture models, GMM) on functional and structural MRI data to identify homogeneous subgroups with distinct developmental trajectories within a heterogeneous population (See 38 for an excellent tutorial on how to apply these models and how to decide upon the optimal number of classes). These mixture models can actually be conceptualized as an extension of multiplegroup latent growth models (48), in which the grouping variable (e.g., individuals with, compared to without, a certain diagnosis) is unobserved. Within the field of developmental psychopathology these mixture models are often used to investigate whether there are subgroups of individuals who show differential development of a certain type of behavior such as depressive symptoms (49), conduct problems (50), and inattention/hyperactivity (51). This modeling approach can reveal smaller subgroups (i.e., around $5-20 \%$ of the total sample) of individuals who show distinct developmental trajectories. Moreover, mixture models allow for the identification of risk and protective factors that could be relevant in the prediction of an individual's growth trajectory. For example, based on their developmental trajectories we have been able to identify a subgroup of young adolescents at risk for developing externalizing problem behaviors. We found that personality traits could predict why some children developed increasing externalizing behaviors, and others don't (52).

When applying mixture models to MRI longitudinal data, a number of considerations with regard to the validity of the identified subgroups are important. First, it is vital that identified subgroups or clusters are replicated in another dataset to verify their existence (48). Large scale, multi-site longitudinal MRI studies such as the ABCD study are a great opportunity for these types of replication (53). Second, we recommend that researchers examine whether the identified clusters are related to other measured variables in predictable ways (48). For instance, prior longitudinal work investigated daily identity formation 
processes in adolescence. It was found that a subgroup of adolescents showed unique developmental trajectories that characterized an adolescent identity crisis (54). This subgroup differed in predictable ways on the development of internalizing and externalizing behaviors as well, which further attests to the substantive meaning of the identified classes (54). Importantly, when using class membership as a predictor of another variable it is possible to use the 'cleanly partitioned subgroups' as categorical predictors. However, mixture models also provide the probability that an individual belongs to each of the identified classes. These class probabilities take into account classification uncertainty, and are therefore recommended to use as predictors rather than the actual class membership. Validity checks are especially vital given that mixture models estimate non-normality in observed data. While this non-normality in the distribution of observed data can actually exist, it may also result from unreliable measurement (48). Despite these possible limitations of mixture models, the field of psychiatry research becomes increasingly interested in analyses to subtype individuals (55-57), such as confirmatory mixture models or more exploratory hybrid machine learning techniques (58) to identify heterogeneity within mental health disorders. Similarly, we believe that future studies could apply latent growth models and mixture models on MRI longitudinal data to examine heterogeneity in brain development as well.

When developmental cognitive neuroscientists are interested in testing for heterogeneity in developmental trajectories they could start with running a univariate latent growth model to test for the general developmental pattern and whether there is significant variability (i.e., a significant random slope) in this average pattern of growth across time (41). If there is indeed significant variability, researchers could proceed with applying mixture models (e.g., latent class growth analyses) to further examine whether, and how many different clusters of individuals can be identified with distinguishable starting points (i.e., intercepts) and change parameters (i.e., slopes) over time (38). 
While growth curve modeling techniques (i.e., mainly mixed-effects models) have been applied to two wave data, at least three waves are recommended when researchers are interested in reliably testing for individual differences in change (i.e., random slopes) to predict certain outcomes (59). Indeed, a recent simulation study showed that two waves of data provide unreliable estimates of the random slopes (36). Both single-cohort designs (repeated assessments of the same cohort across multiple waves) or accelerated cohort designs (following multiple cohorts that are sampled at different ages) can be used to apply mixed-effects and latent growth curve models. For example, we have successfully applied latent growth curve models in a three-wave accelerated longitudinal design (45).

While much progress has been made in charting typical neurodevelopmental trajectories with longitudinal MRI studies, individual differences in intra-individual changes have not been well described so far. When predicting a certain outcome from individual differences in brain features is the main goal, one should start with quantifying how much of the variance in brain volume and/or function is at the between-person versus within-person level. For this purpose, the intraclass correlation (ICC) is often used. Specifically, the ICC is a descriptive statistic that can be used to quantify the variance that is between individuals and the variance over time (60). The ICC can range between 0 and 1 . At the extremes, an ICC of 1 indicates that all the variability in brain volume/activation is between persons, with no within-person variability. In contrast, an ICC of 0 would indicate that there is only withinperson variability across brain assessments and no systematic between-person variability. When researchers want to estimate, for instance the ICC for prefrontal grey matter volume (PFC) over time, they should start with conducting an intercept-only longitudinal multilevel model. This intercept-only model estimates a between-person variance component and a within-person variance component. The following equation provides an example for computing the ICC for grey matter PFC: 
$\frac{\sigma^{2} \text { PFC between-person }}{\sigma^{2} \text { PFC between-person }+\sigma_{\text {PFC within-person }}^{2}}$

where $\sigma^{2}$ PFC between-person is the between-person variance component and $\sigma^{2}$ PFC within-person is the within-person variance component.

Typically, longitudinal structural MRI studies report relatively high ICC (often above .70), which may suggest that some of these brain areas have reached a certain level of stability in the population. Our study further illustrates this relative stability between individuals across time points (Figure 3; adapted from Becht et al., 2018); those adolescents with the highest left and right prefrontal cortex volumes at baseline mostly maintained this position relative to other same aged peers throughout the three waves of data collection. This relative stability also suggests that individual differences at baseline could be the strongest predictor of a certain outcome. Consistent with this idea, in the previously described study of structural brain development predicting later identity development, the majority of predictive effects observed regarded individual differences in baseline prefrontal cortex and nucleus accumbens volumes (45). Individual differences in the amount of within-individual change (i.e., random slopes) in these brain areas were only predicting an outcome measure in two instances. That is, those adolescents who showed a less steep decrease in left nucleus accumbens and right prefrontal cortex volume across three waves, developed at stronger sense of identity over time. Generalizing these findings to the psychiatric research field, we may not always need longitudinal data to predict which individuals develop mental health problems. Instead, the relative position of individuals compared to others at baseline, may already capture most relevant predictive information. If this were the case, it would not only allow us to be quicker in identifying individuals at risk, but also save us the time, money, and effort required to collect longitudinal data. However, we will not know if baseline data are 
most informative unless both the baseline measures and longitudinal change measures are included in predictive efforts.

\section{Conclusion}

The aim of this review was to provide an overview of the substantial heterogeneity in longitudinal patterns of brain development. Longitudinal MRI studies illustrate the large amount of individual differences in baseline and growth of brain development. Yet, so far, only a few longitudinal studies have explicitly tested individual-level brain development, as most have mainly reported group level estimates. We highlighted a number of longitudinal studies that have formally tested individual differences in longitudinally-acquired structural and functional MRI datasets. In reviewing these studies, we describe methodological approaches that could be used by developmental cognitive neuroscience researchers to characterize individual differences in the development of the brain, and identify metrics that could prove useful in predicting why some individuals are at risk of developing mental health disorders.

\section{Acknowledgements and Disclosures}

Kathryn L. Mills was supported by the National Institutes of Mental Health R25MH120869 (PI: Mills). Andrik I. Becht was supported by the Netherlands Organisation for Scientific Research NWO-VICI grant 453-14-001 (PI: Crone) and the Netherlands Organisation for Scientific Research to the Consortium Individual Development grant 024.001.003. Authors report no financial interests or potential conflicts of interest. 


\section{References}

1. Kessler RC, Amminger GP, Aguilar-Gaxiola S, Alonso J, Lee S, Ustun TB (2007): Age of onset of mental disorders: A review of recent literature. Curr Opin Psychiatry 20: 359-364.

2. Kessler RC, Berglund P, Demler O, Jin R, Merikangas KR, Walters EE (2005): Lifetime prevalence and age-of-onset distributions of DSM-IV disorders in the National Comorbidity Survey Replication. Arch Gen Psychiatry 62: 593-602.

3. Rosenberg MD, Casey BJ, Holmes AJ (2018): Prediction complements explanation in understanding the developing brain. Nat Commun 9: 589.

4. Lewis DA, Levitt P (2002): Schizophrenia as a Disorder of Neurodevelopment. Annu Rev Neurosci 25: 409-432.

5. Shaw P, Gogtay N, Rapoport J (2010): Childhood psychiatric disorders as anomalies in neurodevelopmental trajectories. Hum Brain Mapp 31: 917-925.

6. Baltes PB, Reese HW, Nesselroade JR (1977): Life-Span Developmental Psychology: Introduction to Research Methods. Hillsdale, NJ, US: Lawrence Erlbaum Associates, Inc.

7. Baltes PB (1987): Theoretical propositions of life-span developmental psychology: On the dynamics between growth and decline. Dev Psychol 23: 611.

8. Kraemer HC, Yesavage JA, Taylor JL, Kupfer D (2000): How Can We Learn About

Developmental Processes From Cross-Sectional Studies, or Can We? Am J Psychiatry 157: $163-171$

9. Sliwinski M, Hoffman L, Hofer SM (2010): Evaluating Convergence of Within-Person Change and Between-Person Age Differences in Age-Heterogeneous Longitudinal Studies. Res Hum Dev $7: 45-60$

10. Mills KL (2019, June 11): The Necessity of Longitudinal Imaging for Characterizing Brain Maturation. https://doi.org/10.5281/zenodo.3244378 
11. Wierenga LM, Langen M, Oranje B, Durston S (2014): Unique developmental trajectories of cortical thickness and surface area. NeuroImage 87: 120-126.

12. Aubert-Broche B, Fonov VS, García-Lorenzo D, Mouiha A, Guizard N, Coupé P, et al. (2013): A new method for structural volume analysis of longitudinal brain MRI data and its application in studying the growth trajectories of anatomical brain structures in childhood. NeuroImage 82: $393-402$.

13. Raznahan A, Shaw P, Lalonde F, Stockman M, Wallace GL, Greenstein D, et al. (2011): How Does Your Cortex Grow? J Neurosci 31: 7174-7177.

14. Vijayakumar N, Allen NB, Youssef G, Dennison M, Yücel M, Simmons JG, Whittle S (2016): Brain development during adolescence: A mixed-longitudinal investigation of cortical thickness, surface area, and volume. Hum Brain Mapp 37: 2027-2038.

15. Mutlu AK, Schneider M, Debbané M, Badoud D, Eliez S, Schaer M (2013): Sex differences in thickness, and folding developments throughout the cortex. NeuroImage 82: 200-207.

16. Vijayakumar N, Mills KL, Alexander-Bloch A, Tamnes CK, Whittle S (2018): Structural brain development: A review of methodological approaches and best practices. Dev Cogn Neurosci 33: $129-148$.

17. Mills KL, Goddings A-L, Herting MM, Meuwese R, Blakemore S-J, Crone EA, et al. (2016): Structural brain development between childhood and adulthood: Convergence across four longitudinal samples. NeuroImage 141: 273-281.

18. Herting MM, Johnson C, Mills KL, Vijayakumar N, Dennison M, Liu C, et al. (2018):

Development of subcortical volumes across adolescence in males and females: A multisample study of longitudinal changes. NeuroImage 172: 194-205.

19. Tamnes CK, Herting MM, Goddings A-L, Meuwese R, Blakemore S-J, Dahl RE, et al. (2017): Development of the Cerebral Cortex across Adolescence: A Multisample Study of InterRelated Longitudinal Changes in Cortical Volume, Surface Area, and Thickness. J Neurosci Off J Soc Neurosci 37: 3402-3412. 
20. Telzer EH, McCormick EM, Peters S, Cosme D, Pfeifer JH, van Duijvenvoorde ACK (2018): Methodological considerations for developmental longitudinal fMRI research. Dev Cogn Neurosci 33: 149-160.

21. Braams BR, van Duijvenvoorde AC, Peper JS, Crone EA (2015): Longitudinal changes in adolescent risk-taking: a comprehensive study of neural responses to rewards, pubertal development, and risk-taking behavior. $J$ Neurosci 35: 7226-7238.

22. Ordaz SJ, Foran W, Velanova K, Luna B (2013): Longitudinal Growth Curves of Brain Function Underlying Inhibitory Control through Adolescence. J Neurosci 33: 18109-18124.

23. Peters S, Van Duijvenvoorde ACK, Koolschijn PCMP, Crone EA (2016): Longitudinal development of frontoparietal activity during feedback learning: Contributions of age, performance, working memory and cortical thickness. Dev Cogn Neurosci 19: 211-222.

24. Anandakumar J, Mills KL, Earl EA, Irwin L, Miranda-Dominguez O, Demeter DV, et al. (2018): Individual differences in functional brain connectivity predict temporal discounting preference in the transition to adolescence. Dev Cogn Neurosci 34: 101-113.

25. Li R, Utevsky AV, Huettel SA, Braams BR, Peters S, Crone EA, van Duijvenvoorde ACK (2019): Developmental Maturation of the Precuneus as a Functional Core of the Default Mode Network. J Cogn Neurosci 1-14.

26. van Duijvenvoorde ACK, Westhoff B, de Vos F, Wierenga LM, Crone EA (2019): A three-wave longitudinal study of subcortical-cortical resting-state connectivity in adolescence: Testing age- and puberty-related changes. Hum Brain Mapp. https://doi.org/10.1002/hbm.24630

27. Dennison M, Whittle S, Yücel M, Vijayakumar N, Kline A, Simmons J, Allen NB (2013): Mapping subcortical brain maturation during adolescence: evidence of hemisphere- and sexspecific longitudinal changes. Dev Sci 16: 772-791.

28. Lebel C, Beaulieu C (2011): Longitudinal Development of Human Brain Wiring Continues from Childhood into Adulthood. J Neurosci 31: 10937-10947. 
29. Zhou D, Lebel C, Treit S, Evans A, Beaulieu C (2015): Accelerated longitudinal cortical thinning in adolescence. NeuroImage 104: 138-145.

30. Dennison M, Whittle S, Yücel M, Vijayakumar N, Kline A, Simmons J, Allen NB (2013):

Mapping subcortical brain maturation during adolescence: evidence of hemisphere- and sexspecific longitudinal changes. Dev Sci 16: 772-791.

31. Vijayakumar N, Allen NB, Youssef G, Dennison M, Yücel M, Simmons JG, Whittle S (2016):

Brain development during adolescence: A mixed-longitudinal investigation of cortical thickness, surface area, and volume. Hum Brain Mapp. https://doi.org/10.1002/hbm.23154

32. Braams BR, Crone EA (2017): Longitudinal Changes in Social Brain Development: Processing Outcomes for Friend and Self. Child Dev 88: 1952-1965.

33. Peters S, Peper JS, Duijvenvoorde ACKV, Braams BR, Crone EA (2017): Amygdalaorbitofrontal connectivity predicts alcohol use two years later: a longitudinal neuroimaging study on alcohol use in adolescence. Dev Sci 20: e12448.

34. Peters S, Van Duijvenvoorde ACK, Koolschijn PCMP, Crone EA (2016): Longitudinal development of frontoparietal activity during feedback learning: Contributions of age, performance, working memory and cortical thickness. Dev Cogn Neurosci 19: 211-222.

35. Hertzog C, Oertzen T von, Ghisletta P, Lindenberger U (2008): Evaluating the Power of Latent Growth Curve Models to Detect Individual Differences in Change. Struct Equ Model Multidiscip J 15: 541-563.

36. King KM, Littlefield AK, McCabe CJ, Mills KL, Flournoy J, Chassin L (2018): Longitudinal modeling in developmental neuroimaging research: Common challenges, and solutions from developmental psychology. Dev Cogn Neurosci 33: 54-72.

37. Curran PJ (2003): Have Multilevel Models Been Structural Equation Models All Along? Multivar Behav Res 38: 529-569. 
38. Jung T, Wickrama KAS (2008): An Introduction to Latent Class Growth Analysis and Growth Mixture Modeling. 2: 302-317.

39. Curran PJ, Obeidat K, Losardo D (2010): Twelve Frequently Asked Questions About Growth Curve Modeling. J Cogn Dev 11: 121-136.

40. Galbraith S, Bowden J, Mander A (2017): Accelerated longitudinal designs: an overview of modelling, power, costs and handling missing data. Stat Methods Med Res 26: 374-398.

41. Duncan TE, Duncan SC (2009): The ABC's of LGM: An Introductory Guide to Latent Variable Growth Curve Modeling. Soc Personal Psychol Compass 3: 979-991.

42. Moerbeek M (2011): The effects of the number of cohorts, degree of overlap among cohorts, and frequency of observation on power in accelerated longitudinal designs. Methodology.

43. Tamnes CK, Bos MGN, van de Kamp FC, Peters S, Crone EA (2018): Longitudinal development of hippocampal subregions from childhood to adulthood. Dev Cogn Neurosci 30: 212-222.

44. Bollen KA, Curran PJ (2006): Latent Curve Models: A Structural Equation Perspective, 1 edition. Hoboken, N.J: Wiley-Interscience.

45. Becht AI, Bos MGN, Nelemans SA, Peters S, Vollebergh WA, Branje SJ, et al. (2018): Goaldirected correlates and neurobiological underpinnings of adolescent identity: A multimethod multisample longitudinal approach. Child Dev 89: 823-836.

46. Burrow AL, Hill PL (2011): Purpose as a form of identity capital for positive youth adjustment. Dev Psychol 47: 1196-1206.

47. Nagin DS, Odgers CL (2010): Group-Based Trajectory Modeling in Clinical Research. Annu Rev Clin Psychol 6: 109-138.

48. Ram N, Grimm KJ (2009): Methods and Measures: Growth mixture modeling: A method for identifying differences in longitudinal change among unobserved groups. Int J Behav Dev 33: $565-576$. 
49. Ellis RER, Seal ML, Simmons JG, Whittle S, Schwartz OS, Byrne ML, Allen NB (2017): Longitudinal Trajectories of Depression Symptoms in Adolescence: Psychosocial Risk Factors and Outcomes. Child Psychiatry Hum Dev 48: 554-571.

50. Odgers CL, Moffitt TE, Broadbent JM, Dickson N, Hancox RJ, Harrington H, et al. (2008): Female and male antisocial trajectories: From childhood origins to adult outcomes. Dev Psychopathol 20: 673-716.

51. Jester JM, Nigg JT, Buu A, Puttler LI, Glass JM, Heitzeg MM, et al. (2008): Trajectories of Childhood Aggression and Inattention/Hyperactivity: Differential Effects on Substance Abuse in Adolescence. J Am Acad Child Adolesc Psychiatry 47: 1158-1165.

52. Becht AI, Prinzie P, Deković M, Akker AL van den, Shiner RL (2016): Child personality facets and overreactive parenting as predictors of aggression and rule-breaking trajectories from childhood to adolescence. Dev Psychopathol 28: 399-413.

53. Casey BJ, Cannonier T, Conley MI, Cohen AO, Barch DM, Heitzeg MM, et al. (2018): The Adolescent Brain Cognitive Development (ABCD) study: Imaging acquisition across 21 sites. Dev Cogn Neurosci 32: 43-54.

54. Becht AI, Nelemans SA, Branje SJT, Vollebergh WAM, Koot HM, Denissen JJA, Meeus WHJ (2016): The quest for identity in adolescence: Heterogeneity in daily identity formation and psychosocial adjustment across 5 years. Dev Psychol 52: 2010-2021.

55. Baller EB, Kaczkurkin AN, Sotiras A, Adebimpe A, Bassett DS, Calkins ME, et al. (2019): Neurocognitive and Functional Heterogeneity in Depressed Youth. bioRxiv 778878.

56. Holmes AJ, Patrick LM (2018): The Myth of Optimality in Clinical Neuroscience. Trends Cogn Sci 22: 241-257.

57. Marquand AF, Kia SM, Zabihi M, Wolfers T, Buitelaar JK, Beckmann CF (2019):

Conceptualizing mental disorders as deviations from normative functioning. Mol Psychiatry 24: $1415-1424$. 
58. Feczko E, Miranda-Dominguez O, Marr M, Graham AM, Nigg JT, Fair DA (2019): The Heterogeneity Problem: Approaches to Identify Psychiatric Subtypes. Trends Cogn Sci 23: $584-601$.

59. Rogosa DR, Willett JB (1985): Understanding correlates of change by modeling individual differences in growth. Psychometrika 50: 203-228.

60. Little TD, Schnabel KU, Baumert J rgen (2015): Modeling Longitudinal and Multilevel Data: Practical Issues, Applied Approaches, and Specific Examples. Psychology Press. 
Figure 1. Developmental trajectories and individual differences in brain measures for: (A) Average cortical thickness (adapted from Tamnes et al., 2017); (B) Task-based activation of the left nucleus accumbens (NAcc) during win versus lose conditions (adapted from Braams et al., 2015); and (C) Resting-state functional connectivity between the frontal medial cortex and dorsal anterior cingulate cortex (ACC) (adapted from van Duijvenvoorde et al. 2019). Individual scans are represented by dots in each graph, and each individual is connected by a line. Group level trajectories are visible in bolded lines with shaded $95 \%$ confidence intervals for $\mathrm{A}$ and $\mathrm{C}$.

Figure 2. Individual developmental changes for a sample of participants involved in longitudinal MRI studies at age 10 years. In this figure, each scan is represented by one dot and each individual connected by lines. Two individuals are bolded (in pink), demonstrating different average cortical thickness sizes at age 10 years, but continued change in thickness across adolescence and into young adulthood. Figure adapted from data in Tamnes et al., 2017 and presented by (10).

Figure 3. Observed individual volume (in $\mathrm{mm}^{3}$ ) trajectories and average trajectories for (A) left prefrontal cortical and (B) right prefrontal cortical volume. Shaded areas represent $95 \%$ CI. Raw mean scores for prefrontal cortical volume are displayed. Reproduced with permission from Becht et al. (2018). 


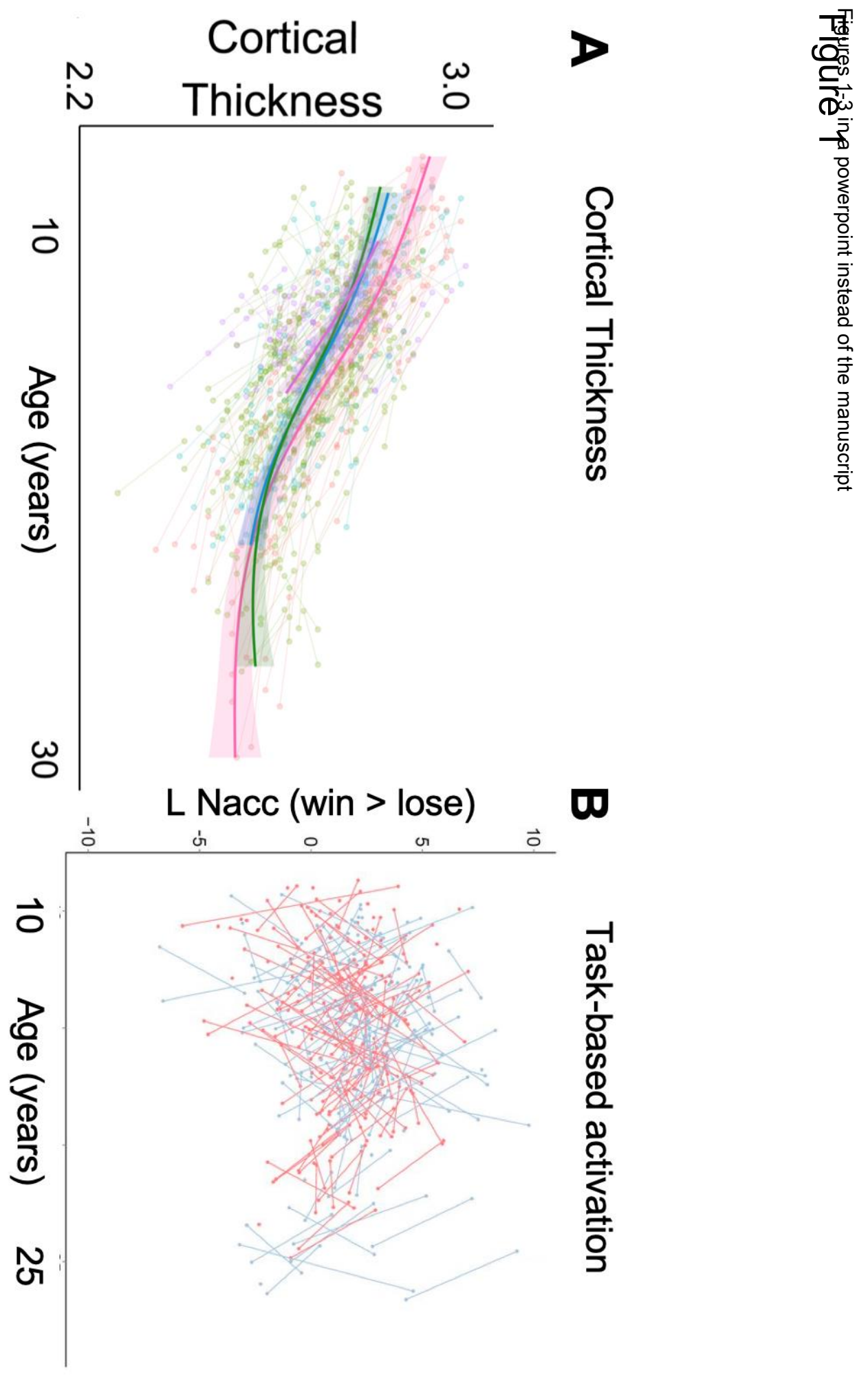

Frontal medial-dorsal ACC $\boldsymbol{\Omega}$
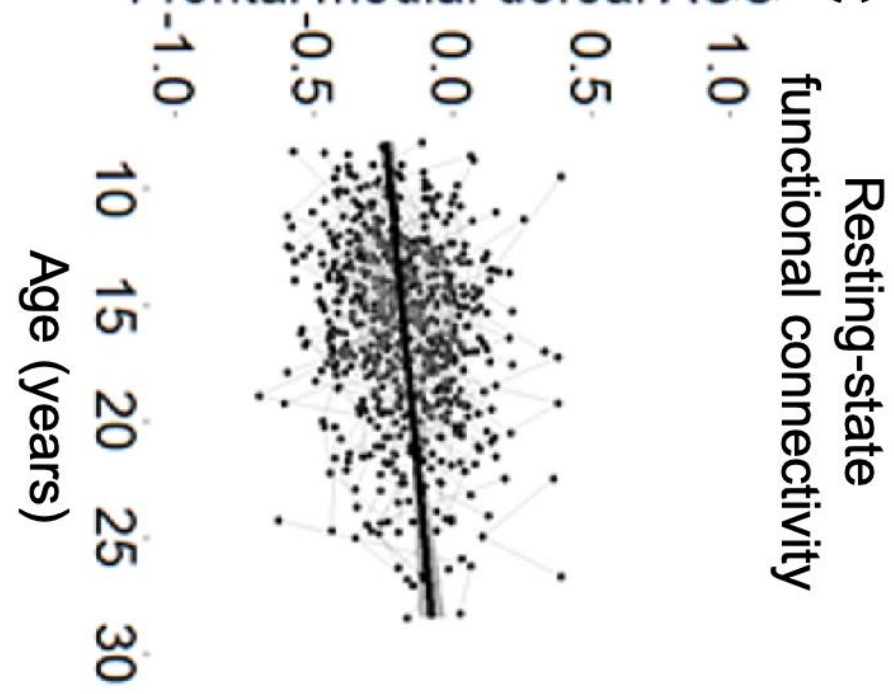
Cortical Thickness

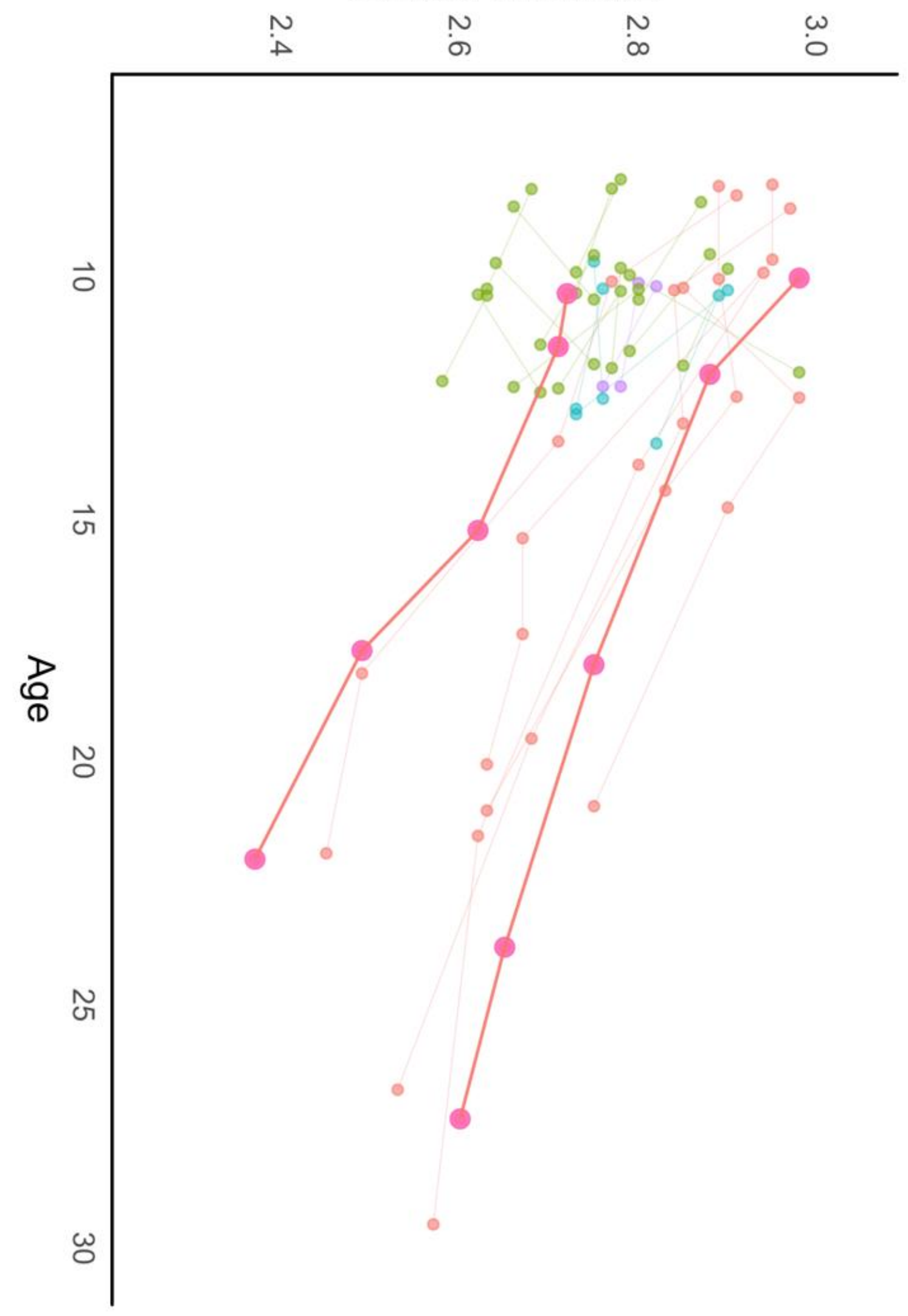




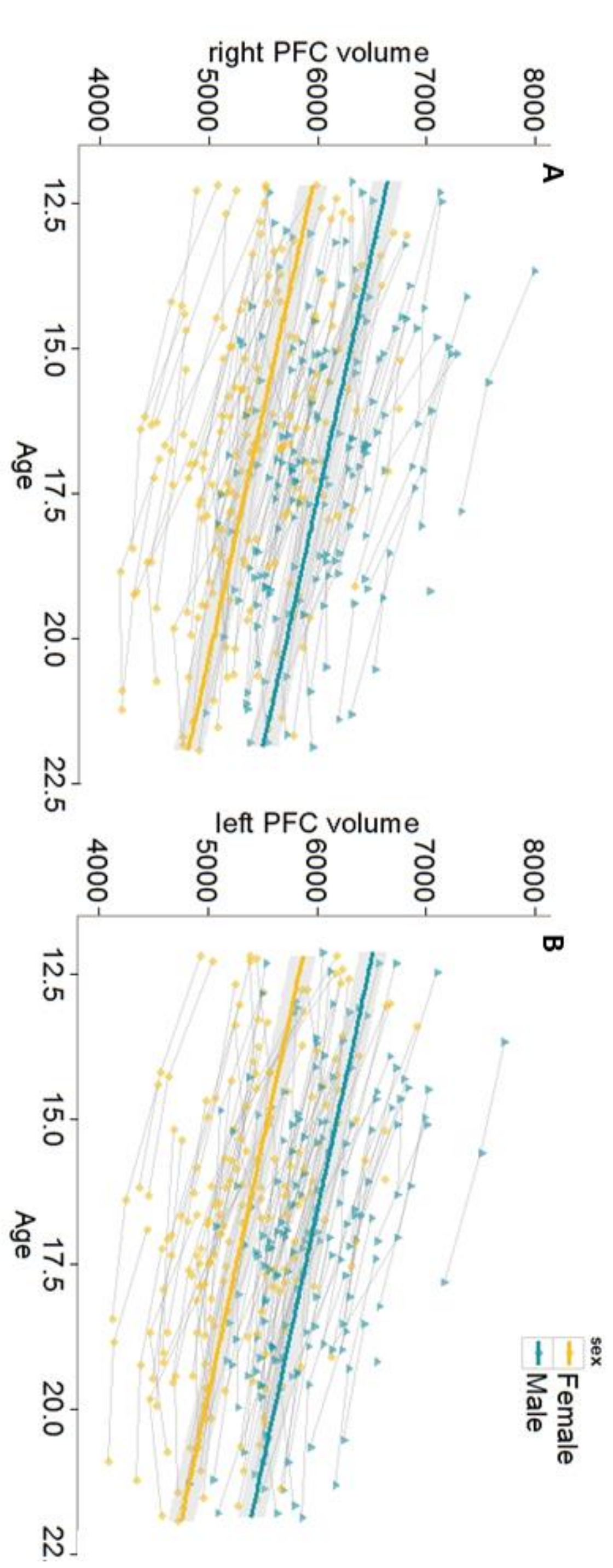

$\frac{\pi}{\frac{0}{C}}$
$\frac{\bar{C}}{\omega}$ 
A major goal of neurodevelopmental research has been to understand how the brain develops in individuals who are diagnosed with mental health disorders, and to use this information for prevention and prediction efforts. In this review, we provide practical methodological recommendations on how to measure individual differences in brain development. 